\title{
Preparando el escenario para dejar de fumar en el paciente con Trastorno Bipolar: intervención breve en la práctica clínica
}

\section{Setting the stage to quit smoking in Bipolar Disorder patients: brief advice in clinical practice}

\begin{abstract}
Fernando Sarramea***, María José Jaén-Moreno***, Vicent Balanzá-Martínez****, María Isabel Osuna*****, José Ángel Alcalá*, Francisco Javier Montiel******, Cristina Gómez******, María Dolores Sánchez******, Ana Belén Rico*, Justa Redondo-Écija*******, Susana Gil*, Francisca Valdivia*, Javier Caballero-Villarraso********, Luis Gutiérrez-Rojas*********.

* Clinical Unit of Mental Health. Reina Sofia University Hospital, Cordoba, Spain. ** Centro de Investigación Biomédica en Red de Salud Mental, CIBERSAM, 33006 Oviedo, Spain. *** Psychiatry section, Faculty of Medicine and Nursing, University of Cordoba, Cordoba, Spain. **** Teaching Unit of Psychiatry and Psychological Medicine, Department of Medicine, University of Valencia, CIBERSAM, Valencia, Spain. Service of Psychiatry, La Fe University and Polytechnic Hospital, Valencia, Spain. ***** Clinical Unit of Mental Health. Virgen de la Victoria University Hospital, Malaga, Spain. ****** Clinical Unit of Mental Health. Complejo Hospitalario de Jaen, Jaen, Spain. ******* Drugs and Addictions Unit. Instituto Provincial Bienestar Social, Diputacion de Cordoba, Cordoba, Spain. ******** Clinical Analyses Service, Reina Sofia University Hospital, Cordoba, Spain. Maimonides Biomedical Research Institute of Cordoba (IMIBIC). ********* Psychiatry and Neurosciences Research Group (CTS-549), Institute of Neurosciences, University of Granada, Granada, Spain.
\end{abstract}

\section{Resumen}

El consumo de tabaco es el principal factor prevenible de mortalidad en pacientes con trastorno bipolar (TB), y las posibles soluciones se encuentran bloqueadas por prejuicios acerca del deseo, posibilidades y riesgos al dejar el consumo de tabaco en estos pacientes. En 8 Centros de Salud Mental se reclutaron consecutivamente pacientes con TB. Los fumadores fueron evaluados antes y después de una intervención breve basada en las 3 As y clasificados según los "estadios de cambio" (EC) y su "disposición para el cambio" (DC). Mediante una regresión lineal múltiple se analizó la evolución del DC y su efecto sobre otras variables independientes (tratamiento farmacológico, historias de síntomas psicóticos, presencia de síntomas de ansiedad, deseo de abandono, capacidad auto-percibida y la percepción subjetiva de funcionamiento cognitivo). Se incluyeron 212 pacientes con TB estabilizados, los fumadores activos $(\mathrm{n}=101 ; 47.6 \%)$ pasaron a la fase de intervención, y un $80.2 \%$ la completaron. Basalmente, $75.2 \%$ consideraban la idea de dejar de fumar, después de la intervención breve, el 30.9\% de los pacientes progresó en su EC. Se observó un incremento significativo del nivel de $\mathrm{DC}$ ( 53.3 vs 59.3, $\mathrm{P}=0.019$ ). La autopercepción del rendimiento cognitivo $(\beta=-0.35 ; \mathrm{P}=0.002)$, el deseo de abandono $(\beta=0.32 ; \mathrm{P}=0.008)$, la autopercepción de la capacidad para dejar de fumar $(\beta=-0.30 ; \mathrm{P}=0.012)$, la edad del paciente $(\beta=-0.72 ; \mathrm{P}=0.004)$, la edad de inicio del tabaquismo $(\beta=0.48 ; \mathrm{P}=0.022)$ y los años fumando $(\beta=0.48 ; \mathrm{P}=0.025)$ fueron $\operatorname{los}$ factores que influyeron significativamente en la posibilidad de cambio tras la intervención breve. Los fumadores con TB consideran la idea de dejar de fumar y una intervención breve desarrollada en el marco de la atención a la salud mental diaria, mejoraría el nivel de preparación. La disfunción neurocognitiva asociada con el TB podría limitar la disposición de los pacientes a dejar de fumar.

Palabras clave: Trastorno bipolar; Tabaco; Abandono tabaquismo; Intervención breve; Disposición para el cambio.

\begin{abstract}
Tobacco consumption is the main preventable factor of mortality in smokers with bipolar disorder (BD), and any possible solutions are often blocked by prejudices over desire, and the possibilities and risks for these patients in giving up tobacco consumption. Adults with BD were recruited at 8 Mental Health Centres. Smokers were evaluated before and after a brief intervention based on the 3 A's and classified into a 'Stage of Change' (SOC) and their 'Readiness to Change' (RTC). A multiple linear regression was used to analyze the progression in their RTC and the independent effect of different variables (pharmacological treatment, history of psychotic symptoms, current anxiety symptoms, willingness, self-perceived capacity to quit smoking and subjective perception of cognitive functioning). Of 212 stable patients diagnosed with $\mathrm{BD}$, current smokers $(\mathrm{n}=101 ; 47.6 \%)$ were included in the intervention phase, and $80.2 \%$ completed it. At baseline, $75.2 \%$ were considering the idea of giving up smoking and, after the brief intervention, $30.9 \%$ of the patients progressed in their SOC. A significant increase in the level of RTC was observed (53.3 vs 59.3, $\mathrm{P}=0.019)$. Perception of cognitive performance $(\beta=-$ $0.35 ; \mathrm{P}=0.002)$, the degree of willing to quit $(\beta=0.32 ; \mathrm{P}=0.008)$, selfperceived capacity to quit tobacco smoking $(\beta=-0.30 ; \mathrm{P}=0.012)$, the patient's age $(\beta=-0.72 ; \mathrm{P}=0.004)$, the age of onset of smoking $(\beta=0.48 ; P=0.022)$ and years as a smoker $(\beta=0.48 ; P=0.025)$ were all factors that significantly influenced the chances of improving after the short intervention. Smokers with BD consider the idea of quitting and a brief intervention developed in the every day mental health care setting improves the level of readiness. The neurocognitive dysfunction associated with BD may limit patients' readiness to quit smoking.

Keywords: Bipolar disorder; Tobacco; Smoking cessation; Brief advice; readiness to change.
\end{abstract}

Recibido: Agosto 2017; Aceptado: Noviembre 2017.

Enviar correspondencia a:

Fernando Sarramea Crespo. Clinical Unit of Mental Health, Reina Sofia University Hospital.

Avda. Menéndez Pidal s/n 14014 Cordoba, Spain. E-mail: fscferro69@gmail.com. 
A ctualmente, los trastornos mentales y la pobreza son las principales áreas de consumo de tabaco en las poblaciones occidentales (Schroeder, 2014). En pacientes con Trastorno Mental grave (TMG), los índices de prevalencia de uso del tabaco son de dos a cuatro veces superiores que en la población general (Díaz et al., 2009). Más de la mitad de los pacientes con trastorno bipolar (TB) fuman (Tidey y Miller, 2015; Jackson, Diaz, Lopez y de Leon, 2015) y el tabaco es la sustancia de abuso que más consumen (George, Wu y Weinberger, 2012). Empiezan a fumar más jóvenes, fuman más intensamente - consumiendo más por día e inhalando más profundamente - y tienen un mayor grado de dependencia de la nicotina (Heffner, Strawn, DelBello, Strakowski yAnthenelli, 2011).

Los mecanismos que pueden explicar esta mayor prevalencia son complejos y pueden implicar una interacción gen-medioambiente (Gonzalez-Pinto et al., 1998). La opción de fumar como una auto-medicación, una teoría comúnmente debatida en pacientes con esquizofrenia y trastornos depresivos (Al-Halabí et al., 2017), al parecer, se puede descartar en el TB (de Leon, Diaz, Aguilar, Jurado y Gurpegui, 2006). Comparado con los no-fumadores con TB, los pacientes con TB que fuman presentan: síntomas más severos, una frecuencia mayor de ciclado rápido, mayor riesgo de suicidio, un mayor número de ingresos hospitalarios (Heffner et al., 2011), peor calidad de vida a nivel mental (Gutiérrez-Rojas et al., 2008) y una tasa de mortalidad cardiovascular y respiratoria que es doble y el triple, respectivamente, que la de la población general (García-Portilla et al., 2010).

El uso del tabaco es el principal factor prevenible de riesgo de mortalidad en esta población (Callaghan et al., 2014), y con el aumento de la evidencia de tratamientos farmacológicos y psicológicos efectivos y seguros (Anthenelli et al., 2016; García-Portilla et al., 2016; Almadana et al., 2017), la literatura destaca la necesidad de abordar este problema en la atención psiquiátrica diaria (Prochaska et al., 2011).

Conocer la disposición de los sujetos a cambiar y tomar medidas para aumentar su motivación y controlar los factores que la limitan son los primeros pasos en cualquier plan de tratamiento del tabaquismo. En el TB, los primeros ensayos en fumadores han comenzado recientemente (Weinberger, Krishnan-Sarin, Mazure y McKee, 2008; Wu et al., 2012; Chengappa et al., 2014; Evins, Cather y Laffer, 2015; Anthenelli et al., 2016; García-Portilla et al., 2016). Estos ensayos incluyen pacientes que han expresado el deseo de dejar de fumar pero no evalúan el nivel de motivación inicial específicamente. Hasta ahora, las principales referencias proceden de estudios en pacientes con esquizofrenia (Etter y Etter, 2004). Weinberguer et al. (2008) y Wu et al. (2012) fueron los primeros en informar de problemas serios de reclutamiento, puesto que no podrían encontrar pacientes bipolares dispuestos a dejar de fumar. Sin embargo, recientemente, miembros de nuestro grupo han publicado los resultados de un tratamiento multi-componente en un contexto clínico (Garcia Portilla et al., 2016), que pone el énfasis principal en la etapa de preparación y motivación, antes de iniciar el tratamiento activo para la esquizofrenia y el TB (Prochaska et al., 2011). Una encuesta online en los E.E.U.U. puso de manifiesto que, de los fumadores con TB que habían realizado un promedio de 4 intentos anteriores, hasta un $74 \%$ expresan la voluntad de dejar de fumar y sólo un tercio había recibido una recomendación de su psiquiatra (Prochaska et al., 2011).

Si bien hay múltiples teorías para explicar el concepto de motivación y cambios en el comportamiento (Miller, 2011), el modelo transteórico (MTT) desarrollado por Prochascka y DiClemente (1983) ha sido el modelo de referencia para las adicciones. El algoritmo del MTT en las Etapas de Cambio (EC) y la Escalera de Contemplación (Biener y Abrams, 1991) han sido las escalas más utilizadas en los pocos estudios que han analizado los niveles de motivación para dejar de fumar en personas con trastornos mentales (Siru, Hulse y Tait, 20094). El MTT ha sido la herramienta más ampliamente utilizada al investigar en cesación tabáquica y reconoce las EC como puntos de partida, tránsito o de llegada en el proceso, al que se puede añadir un valor predictivo de las posibilidades cambio (Chou et al., 2015). A pesar de la popularidad del MTT, el debate sigue abierto acerca de si utilizar la clasificación EC, basada en puntos de corte arbitrarios, o calcular un índice de "disposición para el cambio" (DC) (Ceccarini, Borrello, Pietrobissa, Manzoni y Castelnuovo, 2015).

El éxito en dejar de fumar es proporcional a la intensidad de la intervención (Fiore y Panel, 2008), pero en el primer objetivo de generar motivación, las intervenciones breves utilizando las cinco As (en inglés: Ask, Advise, Assess, Assist and Arrange [Preguntar, Aconsejar, Evaluar, Asistir y Acordar]) y las tres As (Ask, Advise, y Assess, [Preguntar, Aconsejar y Evaluar]) han demostrado ser rentables en población general (Rice y Stead, 2008) y han recibido el apoyo de los estándares de la práctica clínica (West, McNeill y Raw, 2000). La orientación de la EPA (European Psychiatry Association) sobre estrategias para dejar de fumar (Rüther et al., 2014) recomienda probar estas intervenciones en fumadores con enfermedad mental. En pacientes con TMG, hasta ahora sólo DiClemente et al. (2011) han evaluado la intervención con las cinco As, y señalan la importancia del número de repeticiones de las primeras tres As para las posibilidades a largo plazo de éxito en las EC (DiClemente et al., 2011). En pacientes con TB, si bien se desconocen las posibilidades de la intervención breve, quizás el primer objetivo de la intervención de las tres As sería intentar incrementar el nivel de preparación del fumador a través de los diferentes estadios de motivación para el cambio (precontemplación, contemplación y preparación). 
Por otro lado, el TB se asocia a persistentes déficits neurocognitivos en los amplios dominios de la atención sostenida, la memoria verbal y la cognición prefrontal/ejecutiva, que a su vez son importantes predictores de los resultados funcionales de los pacientes (Vieta et al., 2013). Además, el estatus neuropsicológico se relaciona con la toma de decisiones y actitudes tales como la motivación para cambiar el estilo de vida: La disfunción neurocognitiva puede, por lo tanto, disminuir las posibilidades de progreso al nivel motivacional (Baune y Malhi, 2015). De la misma manera, los síntomas de ansiedad presentes en más del $50 \%$ de los pacientes con TB (Goes, 2015), entre otros factores que incrementan las adicciones comórbidas (Cazard y Ferreri, 2013), empeoran el pronóstico, requieren tratamiento específico y podrían influenciar en las posibilidades de esto tipo de intervención.

El ambiente de cuidados comunitarios disponible para estas poblaciones ofrece ventajas a través de la frecuencia de los contactos, la credibilidad de los profesionales de la salud y el asesoramiento regular que proveen. A pesar de estas ventajas, nos encontramos ante un serio problema de salud y las intervenciones rentables como la intervención breve de las 3 As podría servir para identificar el nivel de motivación y elevar la voluntad inicial para dejar de fumar entre fumadores con TB.

\section{Objetivos del estudio}

1. Describir y cuantificar la prevalencia de tabaquismo y cómo se maneja actualmente en una muestra representativa de pacientes con TB estudiados en un contexto comunitario;

2. conocer el nivel de motivación en el que se encuentra el paciente bipolar fumador, basado en el MTT;

3. evaluar la efectividad de la intervención breve de las tres As (Preguntar, Aconsejar y Evaluar) para aumentar el nivel de motivación del paciente; e

4.identificar las variables socio-demográficas, clínicas o tabáquicas que pueden influir en las posibilidades de éxito de la intervención.

\section{Métodos}

\section{Diseño y configuración}

Se trata de un estudio multi-centro que se llevó a cabo siguiendo un diseño mixto, incluyendo:

1. un estudio trasversal, observacional y descriptivo realizado en la visita basal y

2. un diseño post-intervención, en el que los pacientes que fueron identificadas como fumadores en la visita basal recibieron una intervención breve anti-tabaco en tres sesiones.

La duración del estudio fue de 6 meses y fue realizado en 8 Centros de Salud Mental de 4 provincias de Andalucía, en el sur de España (Córdoba, Granada, Jaén y Mála- ga). El estudio recibió la aprobación del Comité de Ética de la Investigación del Hospital Universitario Reina Sofía de Córdoba.

\section{Participantes}

El reclutamiento se llevó a cabo consecutivamente en conformidad con los criterios CIE-10 (1992) al asistir a las citas de revisión programadas, entre los pacientes adultos con TB (18-65 años) clínicamente estables que acudían a sus citas de revisión programada. La estabilidad clínica y la ausencia de conducta suicida en los 6 meses antes de comenzar el estudio fueron evaluadas por el psiquiatra regular del paciente. Todos ellos firmaron un consentimiento informado para que sus datos pudieran ser utilizados en la base de datos del estudio.

Además, los pacientes que se encontraban en consumo de tabaco activo (desde 1 cigarro/día) en la cita de reclutamiento fueron invitados a participar en la evaluación pre- y post-intervención (Figura 1).

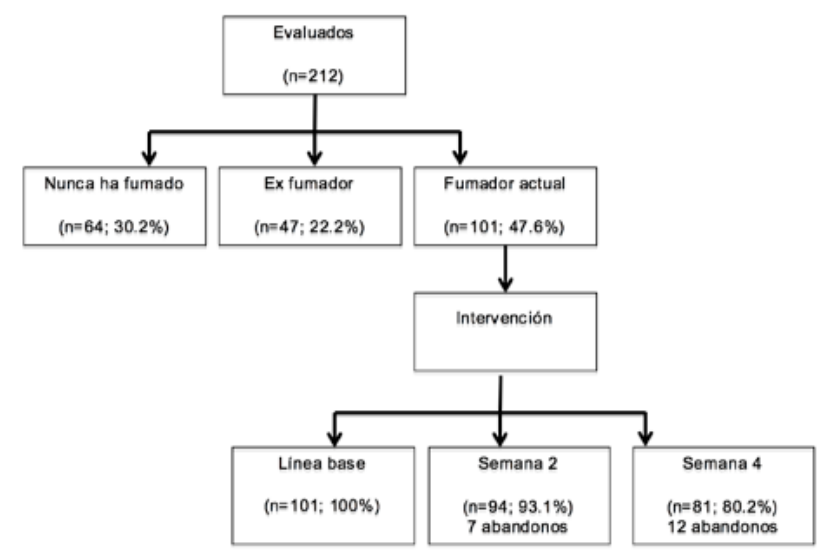

Fig. 1. Reclutamiento de participantes y disposición después de la intervención.

\section{Medidas y resultados}

La muestra total se subdividió en 3 grupos según su hábito tabáquico (fumador activo, exfumador y nunca-fumador). Se recogieron los datos socio-demográficos y clínicos de todos ellos.

Los fumadores activos fueron evaluados utilizando los siguientes instrumentos en la visita basal:

La Hamilton Anxiety Scale (HARS; Hamilton, 1959) se utilizó para determinar si el paciente presentaba síntomas de ansiedad. Esta escala es administrada por un clínico después de una entrevista en que se asignan de 0 a 4 puntos para cada ítem, que evalúan tanto la intensidad como la frecuencia de los síntomas de ansiedad. No hay puntos de corte, y una puntuación más alta indica una mayor intensidad de ansiedad.

Fagerström's Nicotine Dependence Test (FTND; Fageström, 1978) la versión modificada de esta escala (Heatherton, Kozlowski, Frecjer y Fageström, 1991) es hetero-administrado y consiste en 6 ítems que evalúan la dependencia 
a la nicotina. Las puntuaciones se dividen en: menos de 4 (baja dependencia a la nicotina), 4 - 7 (dependencia moderada) y más de 7 (alta dependencia).

Se utilizó una escala visual analógica (EVA) de 0 a 10 para medir el deseo percibido y la capacidad auto-percibida para dejar de fumar, debido a su valor predictiva equivalente a la de escalas con múltiples ítems (Gwaltney, Metrik, Kahler y Shiffman, 2009).

La URICA (University of Rhode Island Change Assessment) (Di Clemente et al., 1991) es un autoinforme de 32 ítems que incluye 4 subescalas y mide el estadío de cambio (EC) basado en el MTT de Prochascka. Los ítems se responden en una escala Likert de 5 puntos que va desde 1 (totalmente en desacuerdo) a 5 (totalmente de acuerdo). La versión española de la escala (Gómez-Peña et al., 2011) ha demostrado adecuada consistencia interna, tanto globalmente como en cada una de las 4 subescalas (entre 0,69 y $0,89)$. Se han descritos dos posibles formas de evaluación: (a) medidas discretas donde la puntuación más alta clasifica al paciente en un EC particular y (b) un continuo en el que el se combinan aritméticamente las subescalas (Contemplación + Acción + Mantenimiento - Precontemplación) para producir una puntuación continua de segundo orden que se puede utilizar para valorar la disposición al cambio (DC). Los participantes completaron la URICA como una forma de llevar a cabo la tercera A ("Assess" - Evaluar) en la visita basal (URICA 1) y en la semana 4 (URICA 2), proporcionando así dos mediciones continuas para la DC. La diferencia entre las dos era la principal variable del análisis $\left(\mathrm{DC}_{2-1}\right)$.

La COBRA, disfunción cognitiva en el trastorno bipolar" (Rosa et al., 2013), es un cuestionario de 16 ítems que mide en los pacientes con TB su percepción de déficits cognitivos en varias áreas, tales como: funciones ejecutivas, velocidad de procesamiento, memoria de trabajo, aprendizaje y recuerdo verbal y atención/concentración. Las respuestas se dan una escala Likert de 3 puntos que van desde 0 (nunca) a 3 (siempre). La puntuación total es el resultado del suma de los ítems. Hemos utilizado la versión española, con un alfa de Cronbach de 0,913.

\section{Intervención}

Se llevó a cabo una intervención breve anti-tabaco basada en los principios de las 3As ("Ask" -Preguntar ¿Fumas?; "Advise" - Aconsejar al paciente que deje de fumar de una manera clara, firme y personalizada; “Assess"- Evaluar ¿Estaría dispuesto a dejar de fumar el mes que viene?) mencionadas antes (Rice y Stead, 2008), distribuida en 3 sesiones (basal, semana 2 y semana 4) de no más de 10 minutos cada una y con un duración total de 30 minutos, la primera y la última presenciales y la de la semana 2 por teléfono. En la visita basal y en la semana 4, se completaron las 3 As, usando la URICA para medir la disposición para dejar de fumar, mientras que la entrevis- ta telefónica se utilizó para reforzar los consejos dados en la intervención basal. Para reducir al mínimo cualquier diferencia inter-observador, el mismo profesional (psiquiatra o enfermera) en cada centro llevó a cabo las tres intervenciones.

\section{Análisis estadísticos}

Las pruebas estadísticas se llevaron a cabo utilizando el programa SPSS (versión 15.0). Se compararon las variables dimensionales y las frecuencias mediante pruebas paramétricas o no paramétricas, según cada caso. Las variables continuas se analizaron con análisis de varianza (ANOVA) o pruebas $t$ de Student, y las variables categóricas con chi-cuadrado o la prueba exacta de Fisher. Se expresaron las correlaciones entre variables como mediante métodos parametricos (test de pearson, $r$ ) y no parametricos (test de spearman $r_{\mathrm{s}}$ ) según el tipo de variable estudiada. Se empleó la regresión lineal múltiple, usando el procedimiento condicional hacia atrás, para analizar el efecto independiente de cada variable (tratamiento farmacológico, historia de síntomas psicóticos, síntomas de ansiedad actuales, disposición, capacidad auto-percibida para dejar de fumar y percepción subjetiva de funcionamiento cognitivo), y el cambio en DC después de la intervención. Para ello, se calculó una nueva variable (DC post-intervención menos DC pre-intervención: $\left.\mathrm{DC}_{2-1}\right)$, la variable dependiente fundamental. Los modelos multivariados incluyen las variables estadísticamente significativas o las de especial relevancia clínica en el análisis bivariado. El nivel de significación estadística se fijó en $p<0,05$.

\section{Resultados}

De los 212 pacientes diagnosticados con TB, 101 $(47.6 \%)$ eran fumadores activos y fueron incluidos en la fase de intervención. De los pacientes restantes, 47 eran exfumadores y 64 nunca habían fumado. La Tabla 1 muestra los datos socio-demográficos y clínicos de los tres grupos (fumadores, exfumadores y nunca-fumadores). Al comparar estos tres grupos, encontramos algunas diferencias: El grupo de intervención (fumadores activos) contenía una proporción superior de varones, pacientes separados o divorciados, desempleados o discapacitados y pacientes con trastornos psiquiátricos concomitantes. No encontramos diferencias en cuanto al nivel de educación, duración de la enfermedad, presencia de síntomas psicóticos o tratamiento farmacológico recibido (Tabla 1 ).

81 pacientes $(80,2 \%)$ del grupo de intervención completaron el seguimiento. En todos los casos que no completaron el seguimiento fue por su deseo de abandonar el estudio. No encontramos diferencias estadísticamente significativas entre los que completaron la intervención y los que no en las variables analizadas (datos socio-demográficos, clínicos e historia de fumar, EC, DC, dependencia 
F. Sarramea, M. J. Jaén-Moreno, V. Balanzá-Martínez, M. I. Osuna, J. Á. Alcalá, F. J. Montiel, C. Gómez,

M. D. Sánchez, A. B. Rico, J. Redondo-Écija, S. Gil, F. Valdivia, J. Caballero-Villarraso, L. Gutiérrez-Rojas

Tabla 1. Descripción de la muestra

\begin{tabular}{|c|c|c|c|c|}
\hline & $\begin{array}{l}\text { Fumador activo } \\
(101 ; 47.6 \%)\end{array}$ & $\begin{array}{l}\text { Ex fumador } \\
(47 ; 22.2 \%)\end{array}$ & $\begin{array}{l}\text { Nunca fumador } \\
(64 ; 30.2 \%)\end{array}$ & Test estadístico, $p$ \\
\hline Edad media (SD) & $47,5(9,8)$ & $49,7(8,2)$ & $50,7(11,1)$ & $2,159^{1}, 0,118$ \\
\hline $\begin{array}{l}\text { Género [n (\%)] } \\
\text { Masculino } \\
\text { Femenino }\end{array}$ & $\begin{array}{l}45(56,2) \\
56(42,7)\end{array}$ & $\begin{array}{l}21(26,2) \\
25(19,1)\end{array}$ & $\begin{array}{l}14(17,5) \\
50(38,2)\end{array}$ & $10,056^{2}, \mathbf{0 , 0 0 7}$ \\
\hline $\begin{array}{l}\text { Estado civil [n (\%)] } \\
\text { Nunca se casó } \\
\text { Casado/a o vive con una pareja } \\
\text { Viudo/a o separado/a-divorciado/a }\end{array}$ & $\begin{array}{l}28(54,9) \\
35(36,5) \\
36(61,0)\end{array}$ & $\begin{array}{r}8(15,7) \\
25(26,0) \\
12(20,3)\end{array}$ & $\begin{array}{l}15(29,4) \\
36(37,5) \\
11(18,6)\end{array}$ & $11,295^{2}, \mathbf{0 , 0 2 3}$ \\
\hline $\begin{array}{l}\text { Nivel de estudios [n (\%)] } \\
\text { Sin estudios formales } \\
\text { Escuela primaria } \\
\text { Escuela secundaria } \\
\text { Universidad }\end{array}$ & $\begin{array}{r}8(42,1) \\
41(47,1) \\
33(51,6) \\
18(43,9)\end{array}$ & $\begin{array}{r}2(10,5) \\
17(19,5) \\
15(23,4) \\
13(31,7)\end{array}$ & $\begin{array}{l}9(47,4) \\
29(33,3) \\
16(25,0) \\
10(24,4)\end{array}$ & $6,753^{2}, 0,344$ \\
\hline $\begin{array}{l}\text { Estatus laboral [n }(\%)] \\
\text { Desempleado } \\
\text { Trabaja (tiempo completo/tiempo parcial) } \\
\text { Discapacitado (temporal/permanente) } \\
\text { Otros* }\end{array}$ & $\begin{array}{c}22(51,2) \\
24(55,8) \\
46(51,7) \\
9(24,3)\end{array}$ & $\begin{array}{c}10(23,3) \\
9(20,9) \\
21(23,6) \\
7(18,9)\end{array}$ & $\begin{array}{l}11(25,6) \\
10(23,3) \\
22(24,7) \\
21(56,8)\end{array}$ & $16,074^{2}, \mathbf{0 , 0 1 3}$ \\
\hline $\begin{array}{l}\text { Diagnóstico [n (\%)] } \\
\text { Trastorno bipolar I } \\
\text { Trastorno bipolar II }\end{array}$ & $\begin{array}{l}62(45,3) \\
38(51,4)\end{array}$ & $\begin{array}{l}26(19,0) \\
21(28,4)\end{array}$ & $\begin{array}{l}49(35,8) \\
15(20,3)\end{array}$ & $6,087^{2}, \mathbf{0 , 0 4 8}$ \\
\hline $\begin{array}{l}\text { Trastornos comórbidos [n (\%)] } \\
\text { Trastorno de ansiedad } \\
\text { Trastorno de personalidad } \\
\text { Trastorno por consumo de sustancias }\end{array}$ & $\begin{array}{c}16(55,2) \\
14(60,9) \\
8(72,7)\end{array}$ & $\begin{array}{c}2(6,9) \\
2(8,7) \\
3(27,3)\end{array}$ & $\begin{array}{c}11(37,9) \\
7(30,4) \\
0(0)\end{array}$ & $13,996^{2}, \mathbf{0}, 030$ \\
\hline $\begin{array}{l}\text { Duración de la enfermedad, meses [mediana } \\
\text { (rango)] }\end{array}$ & $162(536)$ & $168(416)$ & $170(468)$ & $0,731^{1}, 0,483$ \\
\hline Síntomas psicóticos, sí [n (\%)] & $68(50,4)$ & $31(23,0)$ & $36(26,7)$ & $2,000^{2}, 0,368$ \\
\hline $\begin{array}{l}\text { Tratamiento, sí [n (\%)] } \\
\text { Estabilizadores del estado emocional } \\
\text { Antipsicóticos } \\
\text { Antidepresivos } \\
\text { Benzodiazepinas } \\
\text { Hipnóticos }\end{array}$ & $\begin{array}{l}88(48,9) \\
75(47,8) \\
37(46,2) \\
53(47,3) \\
19(51,4)\end{array}$ & $\begin{array}{c}37(20,6) \\
35(22,3) \\
16(20,0) \\
21(18,8) \\
7(18,9)\end{array}$ & $\begin{array}{l}55(30,6) \\
47(29,9) \\
27(33,8) \\
38(33,9) \\
11(29,7)\end{array}$ & $\begin{array}{l}2,224^{2}, 0,329 \\
0,050^{2}, 0,975 \\
0,950^{2}, 0,622 \\
2,653^{2}, 0,265 \\
0,381^{2}, 0,827\end{array}$ \\
\hline
\end{tabular}

Nota. *Otros incluye ama de casa, estudiante y jubilado.

1. prueba ANOVA, 2. Prueba Chi-cuadrado

Tabla 2. Fumadores activos, visita basal clínica y características de la conducta de fumar ( $n=81)$

\begin{tabular}{lcc}
\hline & Media & (SD) \\
\hline HAM-A & 13,6 & 10,4 \\
COBRA & 13,0 & 9,5 \\
Auto-informados CPD & 18,1 & 11,2 \\
FNND & 4,9 & 2,6 \\
Deseo de abandono & 6,2 & 3,4 \\
Capacidad auto-percibida para dejar de fumar & 4,7 & 3,1 \\
DC 1 & 53,3 & 25,9 \\
DC 2 & 59,3 & 25,9 \\
DC2-1 & 7,1 & 22,5 \\
Número de intentos & 2,7 & 2,5 \\
Duración media de los intentos en meses & 11,9 & 21,4 \\
\hline
\end{tabular}

Nota. SD: Desviación típica; COBRA: Cognitive complaints in bipolar disorder rating assessment (Valoración de quejas cognitivas en el trastorno bipolar); FTND: Fagerström Test for Nicotine Dependence HAM-A: Hamilton anxiety rating scale. CPD: Cigarrillos por día.
Tabla 3. Regresión lineal múltiple de factores asociados al cambio en la disposición para cambiar después de la intervención $\left(D C_{2.1}\right)$ en fumadores activos $(n=81)$

\begin{tabular}{lccc}
\hline Variables & $\begin{array}{c}\text { Coeficiente de } \\
\text { correlación } \\
\text { parcial ( } \boldsymbol{\beta}) \mathbf{t}_{\text {exp }}\end{array}$ & valor $\boldsymbol{P}$ \\
\hline Disposición para cambiar* & & & \\
Edad (años) & $-0,72$ & $-2,96$ & 0,004 \\
Años fumando & 0,58 & 2,28 & 0,025 \\
Edad al empezar a fumar (años) & 0,48 & 2,34 & 0,022 \\
Deseo de abandono & 0,32 & 2,71 & 0,008 \\
Capacidad auto-percibida para dejar de fumar & $-0,30$ & $-2,57$ & 0,012 \\
Puntuación en la escala de cognición COBRA & $-0,35$ & $-3,28$ & 0,002 \\
\hline
\end{tabular}

Nota. ${ }^{*}$ Coeficiente de determinación ( $\mathrm{R} 2$ ajustado) $=0.16, \mathrm{~F}=3.38, g l=1,74$ $\mathrm{P}=0,003$ 
y nivel de ansiedad, autopercepción de la capacidad para dejar de fumar y función cognitiva).

En aquellos pacientes que eran fumadores activos, al $80,2 \%$ de ellos nunca les habían ofrecido un tratamiento específico para dejar de fumar durante los años de seguimiento en sus centros de salud mental y al $62.1 \%$ nunca se les preguntó sobre sus hábitos de fumar. En el grupo de exfumadores, el $75 \%$ informó que había dejado de fumar sin tratamiento específico y el 81,8\% informó que no hubo ningún empeoramiento clínico de su enfermedad después de dejar de fumar (estas medidas eran autoinformadas).

En el grupo de intervención, la edad media al empezar a fumar fue de 18,9 años $(S D=7,1)$, con un consumo promedio de alrededor de 18,1 cigarrillos por día: $(S D=$ $11,2)$ durante 28,5 años $(S D=10,5)$. El 16,8\% de estos fumadores tenía alta dependencia de nicotina (FTND > 7). El 80,2\% de los fumadores actuales había intentado dejar de fumar, con un promedio de 2,7 intentos $(S D=2,5)$, y había alcanzado un promedio de 11,8 meses sin fumar ( $S D$ $=21,4)$. Las variables clínicas y del uso del tabaco se resumen en la Tabla 2.

En la prueba EVA, los fumadores obtuvieron una calificación de 6.2 en su deseo de abandonar el tabaco $(S D=3,4)$, y de 4,7 en su capacidad auto-percibida de dejar de fumar $(S D=3,1)$. En la evaluación del nivel de motivación en la primera visita mediante la URICA (URICA 1), 17 (16,8\%) de nuestros pacientes estaban en la fase pre-contemplativa, $76(75,2 \%)$ en la fase contemplativa y $8(7,9 \%)$ en la etapa de acción. 30 días después de completar la intervención breve (URICA 2), el 30,9\% de los pacientes había avanzado en su EC; 7 (8,6\%) estaban en la fase pre-contemplativa, 53 $(65,4 \%)$ en la fase contemplativa y $21(25,9 \%)$ en la etapa de acción (Figura 2). La línea base de EC evaluado por la variable cuantitativa continua de la "disposición al cambio" (DC) mostró un incremento significativo desde 53,3 al principio, a 59,3 después de completar la intervención $(p=0,019)$.

Un modelo de regresión lineal múltiple con el número de años que el paciente había fumado, la edad de inicio en el consumo de tabaco y el deseo de abandono asociaba

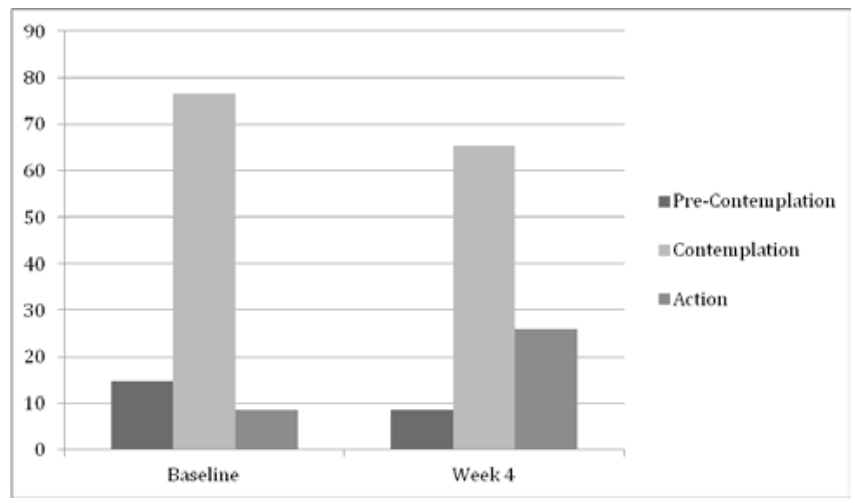

Fig. 2. Estadios motivacionales (antes y después de la intervención) medidos por la escala URICA en el grupo de la intervención (\%). todas estas variables con una mayor evolución de la DC. Además, usando la puntuación total de la escala COBRA, las variables que se asociaban a un menor progreso en la DC fueron la edad, la capacidad auto-percibida para dejar de fumar y el estado cognitivo subjetivo (Tabla 3).

\section{Discusión}

Coincidiendo con resultados anteriores en el TB (Diaz et al., 2009; Tidey y Miller, 2015), encontramos índices de tabaquismo que duplicaron los publicados en población general, y se han confirmado las alertas recurrentes en la literatura acerca de la pasividad del entorno sanitario habitual del paciente (Evins et al., 2015).

Dentro del marco conceptual de los estadios de motivación y el proceso de cambio, y por primera vez en el TB, este estudio de una intervención anti-tabaco llevado a cabo en un contexto comunitario muestra que una intervención de baja intensidad basada en las tres As tiene una repercusión significativa en el nivel de motivación para el cambio en pacientes fumadores-independientemente del grado de consumo de tabaco. La percepción subjetiva del funcionamiento neurocognitivo predice las posibilidades de cambio después de la intervención.

El 30,9\% de los fumadores aumentó su EC y hubo un incremento significativo en el índice DC-ambas medidas se basan en el modelo transteórico del cambio de comportamiento (Prochaska y DiClemente, 1983). Este tipo de intervención, fuertemente apoyada en la literatura para la generación de motivación e intentos de dejar de fumar en población general (Rice y Stead, 2008) apenas se ha estudiado en pacientes con TMG que fuman y, hasta la fecha, no hay trabajos específicos refiriéndose al TB. Di Clemente et al. (2011) informó que hasta un $60 \%$ de pacientes con trastornos psicóticos avanzan en su EC cuando se hace un seguimiento de más de un año, con una intervención planificada usando las cinco As. Además, los pacientes expuestos a repeticiones más frecuentes de las tres primeras As fueron los que más progresaron en esta etapa.

El nivel de deseo de abandono expresado al principio de la intervención, haber empezado a fumar más tardíamente en la vida y haber fumado durante más años fueron las variables que independientemente predijeron un mayor avance en la DC después de la intervención. Estas variables también predicen las posibilidades de un primer intento de abandono en población general (West et al., 2015). Se observó que cuanto más joven fuera el paciente en el momento de la intervención, mayor era la evolución en su nivel de disposición. Esto, en teoría, va en sentido inverso a lo publicado en consejos de salud en la población general, donde las personas mayores tienden a ser más receptivas a este tipo de intervenciones (Zolnierek y Dimatteo, 2009; Marín, Gil y Romero, 2017), pero, sí concuerda con las características favorables de un estudio recientemente 
publicado sobre el primer intento en fumadores (Sharma y Szatkowski, 2014). A pesar de que la población psiquiátrica ha sido menos receptiva a las campañas para reducir el tabaquismo que la población general, los más jóvenesque pueden sentirse menos estigmatizados y han fracasado en menos ocasiones-de hecho, pueden beneficiarse más de ellas y ser más receptivos a esta clase de intervención. Los fumadores que expresaron un mayor nivel de capacidad auto-percibida de cambio al inicio de la intervención mejoraron sus niveles de disposición en un grado menor. Aunque reconozcamos el valor de la capacidad auto-percibida como predictor de intentar dejar de fumar (Borland et al., 2010), suponemos que en un muestra de TB, donde a muchos de los pacientes se les preguntó por primera vez acerca de su uso del tabaco y sus deseos, esa auto-capacidad expresada puede tener un valor poco realista y traducir un bajo insight acerca de la verdadera profundidad de su problema. Esto es similar a la conclusión alcanzada en el meta-análisis de fumadores de la población general (Gwaltney et al., 2009), donde la auto-capacidad se evaluó antes del intento de dejar de fumar.

Una mayor percepción de la limitación funcional al nivel neurocognitivo significa que se podría alcanzar menos progreso en el nivel de preparación. Con respecto a la cognición, estos resultados son difíciles de interpretar, ya que ningún estudio anterior ha examinado el potencial rol del estado neurocognitivo para predecir cambios en la motivación o el dejar de fumar. Se puede extrapolar alguna evidencia indirecta, sin embargo, a partir de estudios de fumadores con esquizofrenia o sin trastornos psiquiátricos. En un conjunto de ensayos clínicos con placebo controlado, la disfunción basal, medida a través de la atención sostenida, la memoria de trabajo espacial y la cognición ejecutiva, se ha asociado al fracaso del tratamiento para el tabaquismo en fumadores con esquizofrenia que buscaban tratamiento (Moss et al., 2009). Subrayamos que la esquizofrenia y el TB comparten un núcleo de disfunción neurocognitiva, con altos índices de dependencia comórbida de la nicotina y bajos índices de abstinencia tabáquica. En otras poblaciones clínicas, las incapacidades cognitivas disminuyeron en respuesta al tratamiento en los sujetos con dependencia de la marihuana o de la cocaína (Aharonovich, Brooks, Nunes y Hasin, 2008). Además, los predictores cognitivos claves para alcanzar la abstinencia en los fumadores sin trastornos psiquiátricos también son la atención sostenida, la memoria de trabajo y el funcionamiento ejecutivo (Ashare, Falcone y Lerman, 2014). Puesto que la corteza prefrontal está implicada en todos estos déficits cognitivos, representa una diana potencial para el desarrollo de terapias farmacológicas y psicológicas encaminadas a tratar déficits cognitivos. A su vez, centrarse en las disfunciones prefrontales puede aumentar los índices de éxito para el abandono tabáquico en todas estas poblaciones y posiblemente también en TB.
En nuestro estudio, la repercusión generada fue independiente del género, el número de cigarrillos por día, y el nivel de dependencia. En los resultados encontrados por DiClemente et al (2011), las mujeres progresaban menos que los hombres, y este resultado también era independiente del consumo de tabaco y de la dependencia. La literatura no dejar lugar a duda sobre el valor pronóstico del nivel de dependencia en los índices de cese inicial y posibles recaídas (Japuntich et al., 2011). Aún así, los resultados que obtuvimos en una muestra con TB parecen coherentes con una intervención diseñada para llevarse a cabo en amplias poblaciones diana, con el objetivo de generar una primera aproximación al nivel de motivación sin especificar el género o la gravedad de la adicción al tabaco (Kruger, O'Halloran, Rosenthal, Babb y Fiore, 2016).

Los resultados de la escala URICA, que posiciona al paciente en un EC o cuantifica un valor DC y su evolución, tienen un valor predictivo con respecto a las posibilidades de un tratamiento diseñado para romper el hábito (Chou et al., 2015). Además, este es el primer estudio que evalúa el EC específicamente en fumadores bipolares. Nuestros resultados confirman que hasta dos terceras partes de los pacientes a quienes se les preguntó sobre su hábito de fumar en un centro ambulatorio se clasifican en la etapa de contemplación, lo que significa que piensan dejar de fumar en los próximos 6 meses y por lo tanto, podrían ser receptores de tratamientos intensivos. Estos resultados son superiores a los descritos en las muestras de pacientes con esquizofrenia (DiClemente et al., 2011) y, como se indicó en el factor edad, sin duda proporcionan una visión perspicaz de la mayor permeabilidad en personas con TB para cambios en el consumo de tabaco que se han producido en los últimos años en población general.

La motivación para el cambio al sufrir una adicción es clave para el reconocimiento del problema y la búsqueda de soluciones. Si bien es urgente saber el nivel de motivación en esta población de fumadores y generar pasos para incrementarlo, saber cómo cuantificar dicho nivel de motivación y su progreso a lo largo del tiempo sigue siendo un reto. Los conceptos de EC y DC y su cuantificación a través de la escala URICA constituyen una opción clave en la investigación de la adicción, pero también tienen sus inconvenientes (DiClemente, Schlundt y Gemmell, 2004). Comprender la motivación desde el EC nos permite posicionar al paciente y proporciona un modelo por el cual podemos observar el proceso de cambio; sin embargo, su cuantificación en estadios discretos o como un continuo también genera debate (DiClemente y Prochaska, 1998). En la adicción al tabaco, una etapa o cifra específica para la preparación no determina un tratamiento específico, pero, como ya hemos destacado, tiene un valor predictivo en el posible éxito del comportamiento del cambio.

Teniendo en cuenta la representatividad de la muestra estudiada y su desarrollo en el ambiente real de cuidados 
comunitarios, nuestro estudio puede ilustrar las posibilidades reales de éxito de una breve intervención para dejar de fumar en pacientes con TB y muestra que puede ser un primer paso simple y rentable que allana el camino para intervenciones más específicas que tendrían como objetivo producir el cambio. En cualquier caso, debemos ser conscientes de la dificultad del problema abordado y se requieren estudios controlados con muestras más grandes como un primer paso para confirmar la efectividad de este tipo de intervención en la práctica clínica, y como una forma de valorar objetivamente la influencia de factores tales como el rendimiento cognitivo. Nosotros evaluamos los factores neurocognitivos a través de un cuestionario validado, el cuestionario COBRA. No obstante, la percepción subjetiva del funcionamiento neurocognitivo no siempre se correlaciona con el rendimiento objetivo en pruebas neuropsicológicas y esto sigue siendo un tema de debate (Miskowiak et al., 2016). Estudios futuros deberían usar baterías neuropsicológicas formales para confirmar o refutar estos resultados.

Por último, identificar los predictores de la motivación/ abstinencia en más estudios de fumadores con TB podría ayudar a diseñar nuevos tratamientos para la adicción a la nicotina. Intervenciones tales como la remediación cognitiva y funcional han demostrado ser eficaces en el TB (Torrent et al., 2013). Además, desde una perspectiva de salud pública, los programas para dejar de fumar dirigidos a pacientes con TB podrían tener en cuenta los potenciales efectos limitantes de la disfunción neurocognitiva para mejorar su motivación para dejar de fumar.

\section{Conflicto de intereses.}

V.B.-M. ha recibido becas y sirvió como consultor, asesor $\mathrm{u}$ orador médico de educación continua (CME) durante los últimos 5 años para las siguientes entidades: Angelini Spain, Angelini Portugal, AstraZeneca, Bristol-Myers-Squibb, Ferrer, Janssen. Juste, Lundbeck, Nutrición Médica, y Otsuka. J.A. Alcalá-Partera ha sido asesor para: Adamed, Lundbeck, Otsuka, Janssen-Cilag, Pfizer, Rovi y Servier; y orador para: Janssen-Cilag, Pfizer y Servier. L. Gutiérrez-Rojas ha sido orador para y miembro del consejo asesor de Bristol-Myers Squibb, Janssen-Cilag, Astra-Zeneca, Rovi, Lundbeck, Otsuka, GSK y Pfizer.

Todos los demás investigadores afirman que no tienen ningún interés biomédico-financiero o posibles conflictos de intereses.

Financiación. Esa investigación no recibió ninguna subvención específica de las agencias de financiación de los sectores público, comercial, o sin fines lucrativos.

\section{Reconocimientos}

Los autores quieren agradecer a los pacientes por participar en este estudio. Agradecemos a V. Millan, M. Ayora,
E. Prieto, M. Toledano, M.J. Notario y P. Zurita por su implicación en la inclusión de los participantes; Agradecemos a F. J. Ayesta, R. Luque, C. Prada y L. Padilla por su ayuda.

\section{Referencias}

Aharonovich, E., Brooks, A. C., Nunes, E .V. y Hasin, D. S. (2008). Cognitive deficits in marijuana users: Effects on motivational enhancement therapy plus cognitive behavioral therapy treatment outcome. Drug and $\mathrm{Al}$ cohol Dependence, 95, 279-283. doi:10.1016/j.drugalcdep.2008.01.009.

Al-Halabí, S., Fernández-Artamendi, S., Díaz-Mesa, E.M., García-Álvarez, L., Flórez, G., Martínez-Santamaría, E.,... Bobes J. (2016). Tobacco and cognitive performance in schizophrenia patients: the design of the COGNICO study. Adicciones, 29, 6-12. doi:10.20882/adicciones.724.

Almadana Pacheco, V., Gómez-Bastero Fernández, A.P., Valido Morales, A., Luque Crespo, E., Monserrat, S. y Montemayor Rubio, T. (2017). Anxiety, depression and tobacco abstinence. Adicciones, 29, 233-244. doi:10.20882/ adicciones.761.

Anthenelli, R. M., Benowitz, N. L., West, R., St Aubin, L., McRae, T., Lawrence, D.,... Evins, A.E. (2016). Neuropsychiatric safety and efficacy of varenicline, bupropion, and nicotine patch in smokers with and without psychiatric disorders (EAGLES): a double-blind, randomised, placebo-controlled clinical trial. Lancet, 387, 2507-2520. doi:10.1016/S0140-6736(16)30272-0.

Ashare, R.L., Falcone, M. y Lerman, C. (2014). Cognitive function during nicotine withdrawal: Implications for nicotine dependence treatment. Neuropharmacology, 76 Pt B, 581-591. doi:10.1016/j.neuropharm.2013.04.034.

Balanzá-Martínez, V., Crespo-Facorro, B., González-Pinto, A. y Vieta, E. (2015). Bipolar disorder comorbid with alcohol use disorder: focus on neurocognitive correlates. Frontiers in Physiology, 6, 108. doi:10.3389/ fphys.2015.00108.

Baune, B.T. y Malhi, G.S. (2015). A review on the impact of cognitive dysfunction on social, occupational, and general functional outcomes in bipolar disorder. Bipolar Disorders, 17 (Suppl 2), 41-55. doi:10.1111/bdi.12341.

Biener, L. y Abrams, D.B. (1991). The Contemplation Ladder: validation of a measure of readiness to consider smoking cessation. Health Psychology, 10, 360-365.

Borland, R., Yong, H.H., Balmford, J., Cooper, J., Cummings, K.M., O'Connor, R.J.,... Fong, G.T. (2010). Motivational factors predict quit attempts but not maintenance of smoking cessation: findings from the International Tobacco Control Four country project. Nicotine and Tobacco Research, 12 Suppl, S4-11. doi:10.1093/ntr/ntq050.

Callaghan, R.C., Veldhuizen, S., Jeysingh, T., Orlan, C., Graham, C., Kakouris, G.,... Gatley, J. (2014). Patterns of tobacco-related mortality among individuals diagno- 
sed with schizophrenia, bipolar disorder, or depression. Journal of Psychiatry Research, 48, 102-110. doi:10.1016/j. jpsychires.2013.09.014.

Cazard, F. y Ferreri, F. (2013). Bipolar disorders and comorbid anxiety: prognostic impact and therapeutic challenges. Encephale 39, 66-74. doi:10.1016/j.encep.2012.04.005.

Ceccarini, M., Borrello, M., Pietrabissa, G., Manzoni, G.M. y Castelnuovo, G. Assessing motivation and readiness to change for weight management and control: an in-depth evaluation of three sets of instruments. Frontiers in Psychology, 6, 511. doi:10.3389/fpsyg.2015.00511.

Chengappa, K.N., Perkins, K.A., Brar, J.S., Schlicht, P.J., Turkin, S.R,... George, T.P. (2014). Varenicline for smoking cessation in bipolar disorder: a randomized, double-blind, placebo-controlled study. Journal Clinical of Psychiatry, 75, 765-772. doi:10.4088/JCP.13m08756.

Chou, K., Chen, H.K., Hung, C.H., Chen, T.T., Chen, C.M. y Wu, B.J. (2015). Readiness to quit as a predictor for outcomes of smoking-reduction programme with transdermal nicotine patch or bupropion in a sample of 308 patients with schizophrenia. European Archives of Psychiatry and Clinical Neuroscience, 265, 249-257. doi:10.1007/ s00406-014-0515-7.

CIE-10., 1992. Trastornos mentales y del comportamiento, descripciones clínicas y pautas para el diagnóstico. Madrid: Meditor.

de Leon, J., Diaz, F.J., Aguilar, M.C., Jurado, D. y Gurpegui, M. (2006). Does smoking reduce akathisia? Testing a narrow version of the self-medication hypothesis. Schizophrenia Research, 86, 256-268. doi:10.1016/j. schres.2006.05.009.

Diaz, F.J., James, D., Botts, S., Maw, L., Susce, M.T. y de Leon, J. (2009). Tobacco smoking behaviors in bipolar disorder: a comparison of the general population, schizophrenia, and major depression. Bipolar Disorders, 11, 154-165. doi:10.1111/j.1399-5618.2009.00664.x.

DiClemente, C.C., Prochaska, J.O., Fairhurst, S.K., Velicer, W.F., Velasquez M.M. y Rossi, J.S. (1991). The process of smoking cessation: An analysis of precontemplation, contemplation, and preparation stages of change. Journal of Consulting and Clinical Psychology, 59, 295-304.

DiClemente, C.C. y Prochascka, J.O. (1998). Toward a comprehensive, transtheoretical model of change: stages of change and addictive behavior. In: Miller WR, Heather N, eds. Treating Addictive Behaviors. 2nd ed. New York, Plenum. Pp: 3-24.

DiClemente, C.C., Schlundt, D. y Gemmell, L. (2004). Readiness and stages of change in addiction treatment. American Journal on Addictions, 13, 103-119. doi:10.1080/10550490490435777.

DiClemente, C.C., Delahanty, J.C., Kofeldt, M.G., Dixon, L., Goldberg, R. y Lucksted, A. (2011). Stage movement following a 5A's intervention in tobacco dependent in- dividuals with serious mental illness (SMI). Addictive Behaviour, 36, 261-264. doi:10.1016/j.addbeh.2010.11.002.

Etter, M. y Etter, J.F. (2004). Alcohol consumption and the CAGE test in outpatients with schizophrenia or schizoaffective disorder and in the general population. Schizophrenia Bulletin, 30, 947-956.

Evins, A.E., Cather, C. y Laffer, A. (2015). Treatment of tobacco use disorders in smokers with serious mental illness; toward clinical best practices. Harvard Review of Psychiatry, 23, 90-98. doi:10.1097/HRP.0000000000000063.

Fagerström, K.O. (1978). Measuring degree of physical dependence to tobacco smoking with reference to individualization of treatment. Addictive Behaviour, 3, 235-241.

Fiore, M. y Panel, G. (2008). Treating tobacco use and dependence: 2008 update. Rockville MD: Department of Health and Human Services.

García-Portilla, M.P., Saiz, P.A., Benabarre, A., Florez, G., Bascaran, M.T., Díaz, E.M.,... Bobes, J. (2010). Impact of substance use on the physical health of patients with bipolar disorder. Acta Psychiatrica Scandinavica, 121, 437445. doi:10.1111/j.1600-0447.2009.01498.x.

García-Portilla, M.P., García-Álvarez, L., Sarramea, F., Galvan, G., Díaz-Mesa, E., Bobes-Bascaran, T.,... Bobes, J. (2016). It is feasible and effective to help patients with severe mental disorders to quit smoking: an ecological pragmatic clinical trial with transdermal nicotine partches and varenicline. Schizophrenia Research, 176, 272280. doi:10.1016/j.schres.2016.05.011.

George, T.P., Wu, B.S. y Weinberger, A.H. (2012). A review of smoking cessation in bipolar disorder: implications for future research. Journal of Dual Diagnosis, 8, 126-130. doi:10.1080/15504263.2012.671717.

Goes, F.S. (2015). The importance of anxiety states in bipolar disorder. Current Psychiatry Reports, 17, 3. doi:10.1007/ s11920-014-0540-2.

Gómez-Peña, M., Penelo, E., Granero, R., Fernández-Aranda, F., Alvarez-Moya, E., Santamaría, J.J.,... Jiménez-Murcia, S. (2011). Motivation to change and pathological gambling: analysis of the relationship with clinical and psychopathological variables. British Journal of Clinical Psychology, 50, 196-210. doi:10.1348/014466510X511006.

Gonzalez-Pinto, A., Gutierrez, M., Ezcurra, J., Aizpuru, F., Mosquera, F., López, P. y de Leon, J. (1998). Tobacco smoking and bipolar disorder. Journal of Clinical Psychiatry, 59, 225-228.

Gutiérrez-Rojas, L., Gurpegui, M., Ayuso-Mateos, J.L., Gutiérrez-Ariza, J.A., Ruiz-Veguilla, M. y Jurado, D. (2008). Quality of life in bipolar disorder patients: a comparison with a general population sample. Bipolar Disorders, 10, 625-634. doi:10.1111/j.1399-5618.2008.00604.x.

Gwaltney, C.J., Metrik, J., Kahler, C.W. y Shiffman, S. (2009). Self-efficacy and smoking cessation: a meta-analysis. Psychol. Addictive Behaviour, 23, 56-66. doi:10.1037/ a0013529. 
Hamilton, M. (1959). The assessment of anxiety states by rating. British Journal of Medical Psychology, 32, 50-55.

Hamilton, M. (1960). A rating scale for depression. Journal of Neurology, Neurosurgery and Psychiatry, 23, 56-62.

Heatherton, T.F., Kozlowski, L.T., Frecjer, R.C. y Fageström, K.O. (1991). The Fageström Test for Nicotine Dependence: A revision of the Fageström Tolerance Questionnaire. British Journal of Addiction, 86, 1119-1127.

Heffner, J.L., Strawn, J.R., DelBello, M.P., Strakowski, S.M. y Anthenelli, R.M. (2011). The co-ocurrence of cigarette smoking and bipolar disorder: phenomenology and treatment considerations. Bipolar Disorders, 13, 439-453. doi:10.1111/j.1399-5618.2011.00943.x.

Jackson, J.G., Diaz, F.J., Lopez, L. y de Leon, J. (2015). A combined analysis of worldwide studies demonstrates an association between bipolar disorder and tobacco smoking behaviors in adults. Bipolar Disorders, 17, 575-597. doi:10.1111/bdi.12319.

Japuntich, S.J., Leventhal, A.M., Piper, M.E., Bolt, D.M., Roberts, L.J., Fiore, M.C. y Baker, T.B. (2011). Smoker characteristics and smoking-cessation milestones. American Journal of Preventive Medicine, 40, 286-294. doi:10.1016/j. amepre.2010.11.016.

Kruger, J., O'Halloran, A., Rosenthal, A.C., Babb, S.D. y Fiore, M.C. (2016). Receipt of evidence-based brief cessation interventions by health professionals and use of cessation assisted treatments among current adult cigarette-only smokers: National Adult Tobacco Survey, 2009-2010. BMC Public Health, 16, 141. doi:10.1186/ s12889-016-2798-2.

Marín Romero, B., Gil Roales-Nieto, J. y Moreno San Pedro, E. (2017). Self-quitting in a Spanish sample. An exploratory study. Adicciones, 29, 210-212. doi:10.20882/ adicciones.925.

Miller, W.R. (2011). Motivational factors in addictive behaviors. In: Miller WR and Carroll KM, (Eds). Rethinking substance abuse: What the science shows and what we should do about it. New York: Guilford Press, pp. 134152.

Miskowiak, K.W., Petersen, J.Z., Ott, C.V., Knorr, U., Kessing, L.V., Gallagher, P. y Robinson, L. (2016). Predictors of the discrepancy between objective and subjective cognition in bipolar disorder: a novel methodology. Acta Psychiatrica Scandinavica, 134, 511-521.

Moss, T.G., Sacco, K.A., Allen, T.M., Weinberger, A.H., Vessicchio, J.C. y George, T.P. (2009). Prefrontal cognitive dysfunction is associated with tobacco dependence treatment failure in smokers with schizophrenia. Drug and Alcohol Dependence, 104, 94-99. doi:10.1111/acps.12649.

Prochaska, J.O. y DiClemente, C.C. (1983). Stages and processes of self-change of smoking: toward an integrative model of change. Journal of Consulting and Clinical Psychology, 51, 390-395.
Prochaska, J.J., Reyes, R.S., Schroeder, S.A., Daniels, A.S., Doederlein, A. y Bergeson, B. (2011). An online survey of tobacco use, intentions to quit, and cessation strategies among people living with bipolar disorder. Bipolar Disorders, 13, 466-473. doi:10.1111/j.13995618.2011.00944.x.

Rhodes, J.D. y Hawk, L.W. JR. (2016). Smoke and mirrors: The overnight abstinence paradigm as an index of disrupted cognitive function. Psychopharmacology, (Berl). 233, 1395-1404. doi:10.1007/s00213-016-4227-8.

Rice, V.H. y Stead, L.F. (2008). Nursing interventions for smoking cessation. Cochrane Database Systematic Review, CD001188. doi:10.1002/14651858.CD001188.pub3.

Rosa, A.R., Mercadé, C., Sánchez-Moreno, J., Solé, B., Mar Bonnin, C.D., Torrent, C.,... Martinez-Aran, A. (2013). Validity and reliability of a rating scale on subjective cognitive deficits in bipolar disorder (COBRA). Journal of Affective Disorders, 150, 29-36. doi:10.1016/j. jad.2013.02.022.

Rüther, T., Bobes, J., De Hert, M., Svensson, T.H., Mann, K., Batra, A.,... Möller, H.J. (2014). EPA guidance on tobacco dependence and strategies for smoking cessation in people with mental illness. European Psychiatry, 29, 6582. doi:10.1016/j.eurpsy.2013.11.002.

Schroeder, S.A. (2014). Even more illness caused by smoking than previously estimated. JAMA Internal Medicine, 174, 1928-1929. doi:10.1001/jamainternmed.2014.4297.

Sharma, A. y Szatkowski, L. (2014). Characteristics of smokers who have never tried to quit: evidence from the British Opinions and Lifestyle Survey. BMC Public Health, 14, 346. doi:10.1186/1471-2458-14-346.

Siru, R., Hulse, G.K. y Tait, R.J. (2009). Assessing motivation to quit smoking in people with mental illness: a review. Addiction, 104, 719-733. doi:10.1111/j.13600443.2009.02545.x.

Tidey, J.W. y Miller, M.E. (2015). Smoking cessation and reduction in people with chronic mental illness. $B M J$, 351, h4065. doi:10.1136/bmj.h4065.

Torrent, C., Bonnin, C del M., Martínez-Arán, A., Valle, J., Amann, B.L., González-Pinto, A.,... Vieta, E. (2013). Efficacy of functional remediation in bipolar disorder: a multicenter randomized controlled study. American Journal of Psychiatry, 170, 852-859. doi:10.1176/appi. ajp.2012.12070971.

Vieta, E., Popovic, D., Rosa, A.R., Solé, B., Grande, I., Frey, B.N.,... Kapczinski, F. (2013). The clinical implications of cognitive impairment and allostatic load in bipolar disorder. European Psychiatry, 28, 21-29. doi:10.1016/j. eurpsy.2011.11.007.

Weinberger, A.H., Krishnan-Sarin, S., Mazure, C.M. y McKee, S.A. (2008). Relationship of perceived risks of smoking cessation to symptoms of withdrawal, craving, and depression during short-term smoking abstinen- 
ce. Addictive Behaviour, 33, 960-963. doi:10.1016/j.addbeh.2008.02.013.

West. R., McNeill, A. y Raw, M. (2000). Smoking cessation guidelines for health professionals: an update. Health Education Authority. Thorax, 55, 987-999. doi:10.1016/j. eurpsy.2011.11.007.

West, R., Raw, M., McNeill, A., Stead, L., Aveyard, P., Bitton, J.,... Borland, R. (2015). Health-care interventions to promote and assist tobacco cessation: a review of efficacy, effectiveness and affordability for use in national guideline development. Addiction, 110, 1388-1403. doi:10.1111/add.12998.

Wu, B.S., Weinberger, A.H., Mancuso, E., Wing, V.C., Haji-Khamneh, B., Levinson, A.J. y George, T.P. (2012). A Preliminary Feasibility Study of Varenicline for Smoking Cessation in Bipolar Disorder. Journal of Dual Diagnosis, 8, 131-132. doi:10.1080/15504263.2012.671067.

Young, R.C., Biggs, J.T., Ziegler, V.E. y Meyer, D.A. (1978). A rating scale for mania: reliability, validity and sensitivity. British Journal of Psychiatry, 133, 429-435.

Zolnierek, K.B. y Dimatteo, M.R. (2009). Physician communication and patient adherence to treatment: a meta-analysis. Medical Care, 47, 826-834. doi:10.1097/MLR. 0b013e31819a5acc. 